\title{
Systematic Reviews and Meta-analysis Published in Indexed Portuguese Medical Journals: Time Trends and Critical Appraisal.
}

\section{Luísa Prada ( $\sim$ luisarussoprada@gmail.com)}

Centro Hospitalar Universitário Lisboa Norte EPE: Centro Hospitalar Universitario Lisboa Norte EPE https://orcid.org/0000-0002-7082-7594

\section{Ana Prada}

Instituto Politecnico de Braganca

\section{Miguel Antunes}

FMUL: Universidade de Lisboa Faculdade de Medicina

\section{Ricardo Fernandes}

Centro Hospitalar Universitário Lisboa Norte EPE: Centro Hospitalar Universitario Lisboa Norte EPE João Costa

Centro Hospitalar Universitário Lisboa Norte EPE: Centro Hospitalar Universitario Lisboa Norte EPE

\section{Joaquim F. Ferreira}

Centro Hospitalar Universitário Lisboa Norte EPE: Centro Hospitalar Universitario Lisboa Norte EPE

\section{Daniel Caldeira}

Centro Hospitalar Lisboa Norte EPE: Centro Hospitalar Universitario Lisboa Norte EPE

\section{Research}

Keywords: AMSTAR II, quality, Systematic review, Meta-analysis, Portugal

Posted Date: March 30th, 2021

DOl: https://doi.org/10.21203/rs.3.rs-354547/v1

License: (c) (i) This work is licensed under a Creative Commons Attribution 4.0 International License. Read Full License

Version of Record: A version of this preprint was published at BMC Medical Research Methodology on April 10th, 2022. See the published version at https://doi.org/10.1186/s12874-022-01591-z. 


\section{Abstract}

\section{Introduction:}

Over the last years, the number of systematic reviews published is steadily increasing due to the global interest in this type of evidence synthesis. However, little is known about the characteristics of this research published in Portuguese medical journals. This study aims to evaluate the publication trends and overall quality of these systematic reviews.

\section{Material and Methods:}

Systematic reviews were identified through an electronic search up to August 2020, targeting Portuguese Medical journals indexed in MEDLINE. Systematic reviews selection and data extraction were done independently by three authors. The overall quality critical appraisal using the A MeaSurement Tool to Assess systematic Reviews (AMSTAR II) was independently assessed by three authors. Disagreements were solved by consensus.

\section{Results:}

Seventy systematic reviews published in 5 Portuguese medical journals were included. Most $(n=57$; $81,4 \%$ ) were systematic reviews without meta-analysis. Until 2010 , the number of systematic reviews per year increased. Since then, the number of reviews published has not remained stable and no less than 3 SRs were published per year. According to the systematic reviews' typology, most have been predominantly conducted to assess the effectiveness of health interventions ( $n=28 ; 40,0 \%)$. General and Internal Medicine ( $n=26 ; 37,1 \%)$ was the most addressed field. Most systematic reviews $(n=45 ; 64,3 \%)$ were rated as being of "critically low-quality".

\section{Conclusions:}

There were consistent flaws in the methodological quality report of the systematic reviews included, particularly in establishing a prior protocol and not assessing the potential impact of the risk of bias on the results.

Through the years, the number of systematic reviews published increased, yet their quality is suboptimal. There is a need to improve the reporting of systematic reviews in Portuguese medical journals, which can be achieved by better adherence to quality checklists/tools.

\section{Systematic review registration: INPLASY202090105}

\section{Background}

Systematic reviews (SRs) and meta-analysis (MA) play an important role in evidence-based clinical practice, since they are thought to produce high-quality evidence that help answering relevant questions 
in a variety of areas in healthcare. Therefore, well-conducted SRs are essential to ensure transparency and to minimize biased information, providing the most valid research evidence on effects of health care interventions $^{(1)}$. In the past few years, the number of SRs and MA publications has significantly increased $(2,3)$, raising concerns about their methodological quality. ${ }^{(4,5)}$ It is well documented that the reliability and validity of SRs' conclusions can be compromised by methodological flaws. ${ }^{(6-8)}$ While this increasing pattern and concerns are known globally, little is known about the characteristics of this type of scientific/clinical research published in Portuguese Medical Journals.

Several tools were developed for undertaking critical appraisal of SRs of healthcare interventions. Currently, one of the most widely used tool for this purpose is AMSTAR II (A MeaSurement Tool to Assess systematic Reviews II). AMSTAR II is the update of AMSTAR, a tool designed for the critical appraisal of SRs of healthcare interventions. While the original tool (AMSTAR), adapted and validated in 2007, includes 11 items, $(9,10)$ AMSTAR II has 16 items in total, allowing for a more detailed assessment of the methodological quality of SRs. ${ }^{(5)}$ Likewise, comparing to the prior version, AMSTAR II also includes SRs based in non-randomized studies, adding to a different scoring system that helps to reduce bias, leading to an overall score. ${ }^{(5,11)}$ Also, unlike the previous tool, AMSTAR II provides a detailed guidance for reviewers. ${ }^{(5)}$ In general, validation studies have shown that the AMSTAR II tool has good measurement properties. ${ }^{(5,9,12)}$

To understand the dynamics and patterns of this growing literature in Portugal, this study aims to evaluate the publication trend, the clinical research field, the typology of SRs, and the overall methodological quality of SRs (assessed using AMSTAR II) published in indexed Portuguese medical journals.

\section{Methods}

\section{PROTOCOL REGISTRATION}

The protocol of this study was developed and registered in the International platform of registered systematic review and meta-analysis protocols (INPLASY) with the following registration number INPLASY202090105 (available at: https://inplasy.com/). The protocol was not published in any peerreviewed journal. Conduct and reporting followed the PRISMA statement ${ }^{(13)}$ (see Additional file 2).

\section{SEARCH METHODS}

Potentially eligible SRs and MA were identified through an electronic search up to August 2020, targeting Portuguese Medical journals indexed in MEDLINE (Search strategy at Additional file 1, table 1).

\section{STUDY SELECTION}


Three of the authors (LP, AP, MMA) independently screened the search results for inclusion, assessing the abstract and then the complete text, according to the inclusion and exclusion criteria. We included SRs, with or without MA, published in Portuguese Medical journals indexed in MEDLINE. We aimed Portuguese Medical Journals indexed to MEDLINE, since we assumed, in a pragmatic approach, that we would find a large number of critically low/low methodological quality SRs in Portuguese Medical journals that are not indexed to MEDLINE.

We adhered to the population, intervention, control, outcome and study design (PICOS) approach for identifying relevant SR and/or MA. SRs were included regardless of research question, methodological or reporting quality, and included study designs. We excluded studies which were not SRs nor MA, as well as SRs or MA that did not included studies enrolling human participants. Conference abstracts were not included, since it is not possible to fully assess their methodological quality based on the information contained in the abstract. Discrepancies were resolved by consensus-based discussion.

\section{DATA EXTRACTION}

For each of the eligible SRs, three reviewers (LP, AP and MMA) independently extracted relevant data into a pre-piloted data collection template and assessed the overall quality of the SRs using AMSTAR II tool. The specific items extracted from the full text were as follows: first author's surname; article's title; journal name; year of publication; type of review conducted (according to Munn et al. criteria $\left.{ }^{(14)}\right)$; predominant research field according to the research category/ classification of Clarivate Analytics (Clarivate Web of Science(C); country and country's region of first author Institution; whether a MA has been conducted or not, and instruments used to evaluate the risk of bias (RoB).

\section{QUALITY ASSESSMENT OF METHODOLOGY}

The methodological quality assessment of the SRs and MA was independently assessed by two reviewers in pairs (LP and AP, MMA and LP) using the AMSTAR II tool. Discrepancies were resolved by consensus-based discussion. Methodological quality assessment is specified in Additional File 1, supplementary data 1.

\section{DATA ANALYSIS}

The collected data were entered and checked in a pre-piloted form. The descriptive statistical analyses included calculations of absolute and relative frequencies of the qualitative variables.

\section{Results}

\section{GENERAL CHARACTERISTICS AND TEMPORAL TRENDS}


We identified and assessed 70 SRs published between 2001 and 2019 (The flowchart in Fig. 1 illustrates the selection process). We identified 5 Portuguese medical journals indexed to MEDLINE. Therefore, 29 (41\%) of SRs included were published in Acta Médica Portuguesa, 16 (22,9\%) in Acta Reumatológica Portuguesa, 12 (17,1\%) in Revista Portuguesa de Cardiologia, 11 (15,\%) in Pulmonology and 2 (2,9\%) in Revista Portuguesa de Cirurgia Cardio-torácica e Vascular. SRs without MA were more frequently published $(n=57 ; 81,4 \%)$ in comparison to SRs with MA $(n=13 ; 18,6 \%)$.

The most frequent research field covered by SRs was General and Internal Medicine (37,1\%). Two $\operatorname{articles}^{(15,16)}(2,9 \%)$ did not have their research category/ classification of Clarivate Analytics available. Table 1 summarises the clinical research fields addressed in the SRs included.

Following the Munn et al. ${ }^{(14)}$ criteria, 28 SRs (40,0\%) were classified as "effectiveness/efficacy", 15 $(21,4 \%)$ as "expert opinion and/or policy", $11(15,7 \%)$ as "prognostic", 8 (11,4\%) as "prevalence" and 5 $(7,1 \%)$ as "diagnostic test accuracy". Very few SRs were categorized as being "costs/economic", "etiology and/or risk" or "evaluation experimental (qualitative)" (Table 2).

According to the country of the first author's institution (Additional file 1, supplementary table 2 and 3 ), the majority were from Portugal $(75,7 \%)$.

Prior to 2010, no more than 2 SRs were published per year. There was a peak in publications in 2010 $(n=11)$ and since then, while the trend has been variable, no less than 3 SRs were published per year (Fig. 2).

\section{METHODOLOGICAL QUALITY}

The overall confidence in the results of 45 SRs $(64,3 \%)$ were rated as "Critically Low quality review", 9 SRs $(12,9 \%)$ were rated as "Low quality" and 15 SRs $(21,4 \%)$ were rated as "Moderate quality" and only one SR (1,4\%) was rated as "High quality" (Fig. 3; see Additional file 1, Supplementary figure 1 and 2 for SRs and quality appraisal using AMSTAR II).

The critical items of AMSTAR II mostly missed in the reporting of SR were: 61 SRs $(87,1 \%)$ did not adhere to a priory well-designed protocol (question 2); 50 SRs (71,4\%) partially reported only some features of a comprehensive literature search (question 4); none of the SRs reported a complete description of the methods used to search for relevant literature; most SRs (64,3\%) did not assess RoB in individual studies that were included in the review (question 9) nor discussed the RoB $(77,1 \%)$ in the results interpretation (questions 13). Nevertheless, among the 13 SRs that performed a MA, 12 SRs (92,3\%) used appropriate methods for the statistical combination of results (question 11) and 9 of the 13 SRs with MA $(69,2 \%)$ conducted a publication bias analyses and discussed its impact (question 15).

The methodological quality of the published SRs has probably improved over time (Fig. 2), showing a decrease of "Critically Low quality" studies, however the low number of published SRs precludes a robust 
evaluation.

Quality according with type of SR is detailed in Additional file 1, supplementary table 4.

\section{Discussion}

To the best of our knowledge, our study is the first to investigate the general characteristics and assess the methodological quality using AMSTAR II tool of SRs and MA published in Portuguese Medical journals indexed in MEDLINE.

The main findings were: the peak of SR publications was in 2010 and there was not a substantial increase of publications during the last decade; The majority of the SRs was classified as being of "Critically Low" quality according to the AMSTAR II tool. Nonetheless, their overall quality has probably increased overtime.

According to our results, while there was a slight increase in the number of SRs publications in the last decade comparing to the prior decade, we did not find it to be a sustained one. This trend was not expectable since SRs are being increasingly published in medical journals globally. ${ }^{(2,17)}$ The potential explanatory reasons include editorial policies and/or authors' choices.

Most of the included SRs were assessed as being of "critically low" overall quality, which is a matter of concern. Few SRs adequately satisfied critical items such as the use of a prior protocol and the assessment of RoB. Establishing a prior well-developed protocol before commencement of the review can reduce the risk of bias and promote transparency in the review process ${ }^{(18,19)}$, ideally through platforms such as International prospective register of systematic reviews (PROSPERO) or the International platform of registered systematic review and meta-analysis protocols (INPLASY). It is also important to highlight that none of the included reviews did a complete description of the methods used to search for relevant literature. This disables the reproducibility of the search, which is one of the crucial foundations for the SRs process. Also, a complete and transparent search process is the best way to avoid publication bias. ${ }^{(20)}$ The absence of a clear description about the details of the literature search strategy is an important flaw. Another concern raised by our analysis is that most of the included studies did not assessed the potential impact of RoB on the results, which is essential to evaluate the validity of the SR results. ${ }^{(20)}$ Furthermore, some authors failed to assess RoB with validated tools, as they developed and used their own tools to do so. ${ }^{(21,22)}$

Other studies have assessed the methodological quality of SRs, with or without MA, published in healthcare/medical journals, using AMSTAR II tool. Most studies report similar results, with a large number of SRs included being rated as low or critical low quality. ${ }^{(23-27)}$

Currently, there are some quality appraisal tools and guidelines that might help authors and peer reviewers to improve and assess methodological report and review of SRs. The AMSTAR II is one of these tools. We used this tool in our study because it allows to categorize $\operatorname{SRs}^{(5,9)}$. There are other tools, such 
as PRISMA ${ }^{(13)}$ or MOOSE ${ }^{(28)}$, which are also extensive tools/checklists that inform and help to improve the quality of the reporting of medical research, but do not have critical items nor categorize the SRs according to their reporting.

We acknowledge that most of the reviews here included are not contemporary of AMSTAR II (nor other instruments that could improve reporting such as PRISMA). However, we would like to claim that the methodological standards for publication in Portuguese medical journals should be high and similar to other European and US journals. We would like to stress the need of editorial policy measures for authors, editors and peer reviewers, like adherence to checklist items and prospective registration of protocols for SRs, in order to strengthen the methodological quality and reliability of SRs/MA published in Portuguese medical journals. ${ }^{(29)}$ As an example, there are journals that require the submission of a methodological checklist filled by authors for editors/reviewer assessment (such as PRISMA), while others started to apply AMSTAR II to screen SRs' methodological quality, in order to promote reviews of higher quality. ${ }^{(30)}$

We also would like to acknowledge that AMSTAR-II has limitations because it is a tool for critical appraisal mostly adapted for interventions/exposures. Also, we were conservative (with less criticism in the evaluation) when appraising non-interventional SRs. The authors of AMSTAR II do not recommend an overall score, despite the electronic version calculates a final score. ${ }^{(5)}$ In fact, we did not use the score directly, instead we used their categorization which we found useful to highlight that the overall quality is low and that publication requirements should be more demanding. Lastly, some of the included reviews, which were classified as being "critically low quality reviews", fail to follow crucial methodological steps in conducting a qualitative and/or quantitative SRs, flaws that call into question its classification as SRs. Given the pedagogical intent and the aim of our study, we opted to include them in the final analysis.

\section{Conclusion}

In the last decade there was an increase of published SRs in indexed Portuguese Medical Journals. Regarding the methodological quality and reporting, most were evaluated as being of "Critically Low quality" according to the AMSTAR II, mainly due to the absence of protocol registration and adequate RoB when interpreting/discussing the results of the review. This call out for a need to improve the reporting of SRs, which can be made by better adherence to quality checklists/tools. Furthermore, specific quality control at the level of journal editors and peer reviewers is warrant, like implementation and adherence to checklist items in order to improve methodological quality of SRs/MA published in Portuguese medical journals.

\section{Abbreviations}


AMSTAR A MeaSurement Tool to Assess systematic Reviews

FMUL Faculdade de Medicina da Universidade de Lisboa

GAPIC Gabinete de Apoio à Investigação Científica

INPLASY International platform of registered systematic review and meta-analysis protocols

MA Meta-analysis

MOOSE Meta-analysis Of Observational Studies in Epidemiology

PRISMA Preferred Reporting Items for Systematic Reviews and Meta-Analyses

RoB Risk of Bias

SR Systematic review

\section{Declarations}

\section{Funding sources}

The author(s) received no financial support for the research, authorship, and/or publication of this article.

\section{Conflicts of interest}

DC in the last 3 years has participated in educational conferences/congresses (including travel, accommodation, and/or hospitality) and has received speaker/consultant fees from Daiichi Sankyo, Menarini, Roche and Merck-Serono. JJF received speaker and consultant fees from GlaxoSmithKline, Novartis, TEVA, Lundbeck, Solvay, Abbott, Bial, Merck-Serono, Grunenthal and Merck Sharp and Dohme. The remaining authors declare no conflicts of interest.

\section{Authors' contributions}

DC is the guarantor and contributed for concept and design. AP, LP and MMA searched the articles and retrieved the data. DC coordinated the data search and retrieval. AP, LP and MMA performed the quality methodological assessment. AP, DC and LP performed the statistical analysis and wrote the first draft. AP, $\mathrm{DC}, \mathrm{LP}$ and MMA were involved in the result interpretation, discussion and text writing. JC, JJF and RF were involved in the analysis and interpretation of the data, critically revised the manuscript for important intellectual content. All the authors approved the version of the manuscript.

\section{Consent for publication}

Not applicable. 


\section{Ethics approval and consent to participate}

Not required as this was a systematic review of publicly available studies

\section{Consent for publication}

Not applicable.

\section{Availability of data and materials}

Not applicable.

\section{Acknowledgements}

Not applicable.

\section{References}

1. Murad MH, Montori VM, loannidis JP, Jaeschke R, Devereaux P, Prasad K, et al. How to read a systematic review and meta-analysis and apply the results to patient care: users' guides to the medical literature. Jama. 2014;312(2):171-9.

2. Riaz IB, Khan MS, Riaz H, Goldberg RJ. Disorganized Systematic Reviews and Meta-analyses: Time to Systematize the Conduct and Publication of These Study Overviews? Am J Med. 2016;129(3):339 e11-8.

3. Bastian H, Glasziou P, Chalmers I. Seventy-five trials and eleven systematic reviews a day: how will we ever keep up? PLoS Med. 2010;7(9):e1000326.

4. Fontelo P, Liu F. A review of recent publication trends from top publishing countries. Syst Rev. 2018;7(1):147.

5. Shea BJ, Reeves BC, Wells G, Thuku M, Hamel C, Moran J, et al. AMSTAR 2: a critical appraisal tool for systematic reviews that include randomised or non-randomised studies of healthcare interventions, or both. BMJ. 2017;358:j4008.

6. Jamshidi L, Heyvaert M, Declercq L, Fernández-Castilla B, Ferron JM, Moeyaert M, et al. Methodological quality of meta-analyses of single-case experimental studies. Research in Developmental Disabilities. 2018;79:97-115.

7. Delaney A, Bagshaw SM, Ferland A, Laupland K, Manns B, Doig C. The quality of reports of critical care meta-analyses in the Cochrane Database of Systematic Reviews: an independent appraisal. Critical care medicine. 2007;35(2):589-94. 
8. Delaney A, Bagshaw SM, Ferland A, Manns B, Laupland KB, Doig CJ. A systematic evaluation of the quality of meta-analyses in the critical care literature. Critical care. 2005;9(5):R575.

9. Shea BJ, Hamel C, Wells GA, Bouter LM, Kristjansson E, Grimshaw J, et al. AMSTAR is a reliable and valid measurement tool to assess the methodological quality of systematic reviews. J Clin Epidemiol. 2009;62(10):1013-20.

10. Shea BJ, Bouter LM, Peterson J, Boers M, Andersson N, Ortiz Z, et al. External validation of a measurement tool to assess systematic reviews (AMSTAR). PLoS One. 2007;2(12):e1350.

11. Greenland S, O'rourke K. On the bias produced by quality scores in meta-analysis, and a hierarchical view of proposed solutions. Biostatistics. 2001;2(4):463-71.

12. Lorenz RC, Matthias K, Pieper D, Wegewitz U, Morche J, Nocon M, et al. A psychometric study found AMSTAR 2 to be a valid and moderately reliable appraisal tool. J Clin Epidemiol. 2019;114:133-40.

13. Liberati A, Altman DG, Tetzlaff J, Mulrow C, Gøtzsche PC, loannidis JP, et al. The PRISMA statement for reporting systematic reviews and meta-analyses of studies that evaluate health care interventions: explanation and elaboration. Journal of clinical epidemiology. 2009;62(10):e1-e34.

14. Munn Z, Stern C, Aromataris E, Lockwood C, Jordan Z. What kind of systematic review should I conduct? A proposed typology and guidance for systematic reviewers in the medical and health sciences. BMC Med Res Methodol. 2018;18(1):5.

15. da Silva Melo E, Casanova J. Cyanotic congenital heart diseases duct dependent: Stent versus surgical shunt. Meta-analysis of observational studies. Revista portuguesa de cirurgia cardiotoracica e vascular: orgao oficial da Sociedade Portuguesa de Cirurgia Cardio-Toracica e Vascular. 2015;22(3):135-44.

16. de Oliveira Pacheco J, Santos J, Moreira SS. Complications of Hypogastric Arteries Embolization in EVAR. Revista portuguesa de cirurgia cardio-toracica e vascular: orgao oficial da Sociedade Portuguesa de Cirurgia Cardio-Toracica e Vascular. 2015;22(1):41-6.

17. Ioannidis JP. The Mass Production of Redundant, Misleading, and Conflicted Systematic Reviews and Meta-analyses. The Milbank quarterly. 2016;94(3):485-514.

18. Silagy CA, Middleton P, Hopewell S. Publishing protocols of systematic reviews: comparing what was done to what was planned. Jama. 2002;287(21):2831-4.

19. Stewart L, Moher D, Shekelle P. Why prospective registration of systematic reviews makes sense. BioMed Central; 2012.

20. Higgins JP, Thomas J, Chandler J, Cumpston M, Li T, Page MJ, et al. Cochrane handbook for systematic reviews of interventions: John Wiley \& Sons; 2019.

21. Lopes C, Pereira T, Barra S. Cardiac resynchronization therapy in patients with atrial fibrillation: $A$ meta-analysis. Revista Portuguesa de Cardiologia (English Edition). 2014;33(11):717-25.

22. Andrade JS, Souza W, Paranhos LR, Domenis DR, Cesar C. [Effects of Speech Therapy in Hospitalized Patients with Post-Stroke Dysphagia: A Systematic Review of Observational Studies]. Acta medica Portuguesa. 2017;30(12):870-81. 
23. Ding M, Soderberg L, Jung JH, Dahm P. Low Methodological Quality of Systematic Reviews Published in the Urological Literature (2016-2018). Urology. 2020.

24. Habtewold TD, Alemu SM, Mohammed SH, Endalamaw A, Mohammed MA, Tefera AA, et al. Overview of biomedical and public health reviews in Ethiopia from 1970 to 2018: trends, methodological qualities, gaps and future directions. bioRxiv. 2018:405555.

25. O'Kelly F, DeCotiis K, Aditya I, Braga L, Koyle M. Assessing the methodological and reporting quality of clinical systematic reviews and meta-analyses in paediatric urology: can practices on contemporary highest levels of evidence be built? Journal of pediatric urology. 2020;16(2):207-17.

26. Leclercq V, Beaudart $C$, Ajamieh $S$, Tirelli $E$, Bruyère $O$. Methodological quality of meta-analyses indexed in PsycINFO: leads for enhancements: a meta-epidemiological study. BMJ open. 2020;10(8):e036349.

27. Dettori JR, Skelly AC, Brodt ED. Critically Low Confidence in the Results Produced by Spine Surgery Systematic Reviews: An AMSTAR-2 Evaluation From 4 Spine Journals. Global Spine Journal. 2020:2192568220917926.

28. Stroup DF, Berlin JA, Morton SC, Olkin I, Williamson GD, Rennie D, et al. Meta-analysis of observational studies in epidemiology: a proposal for reporting. Jama. 2000;283(15):2008-12.

29. Moher D, Shamseer L, Clarke M, Ghersi D, Liberati A, Petticrew M, et al. Preferred reporting items for systematic review and meta-analysis protocols (PRISMA-P) 2015 statement. Systematic reviews. 2015;4(1):1.

30. Jung JH, Dahm P. Reaching for the stars-rating the quality of systematic reviews with the Assessment of Multiple Systematic Reviews (AMSTAR) 2. BJU international. 2018;122(5):717-8.

\section{Tables}

Due to technical limitations, tables are only available as a download in the Supplemental Files section.

\section{Figures}



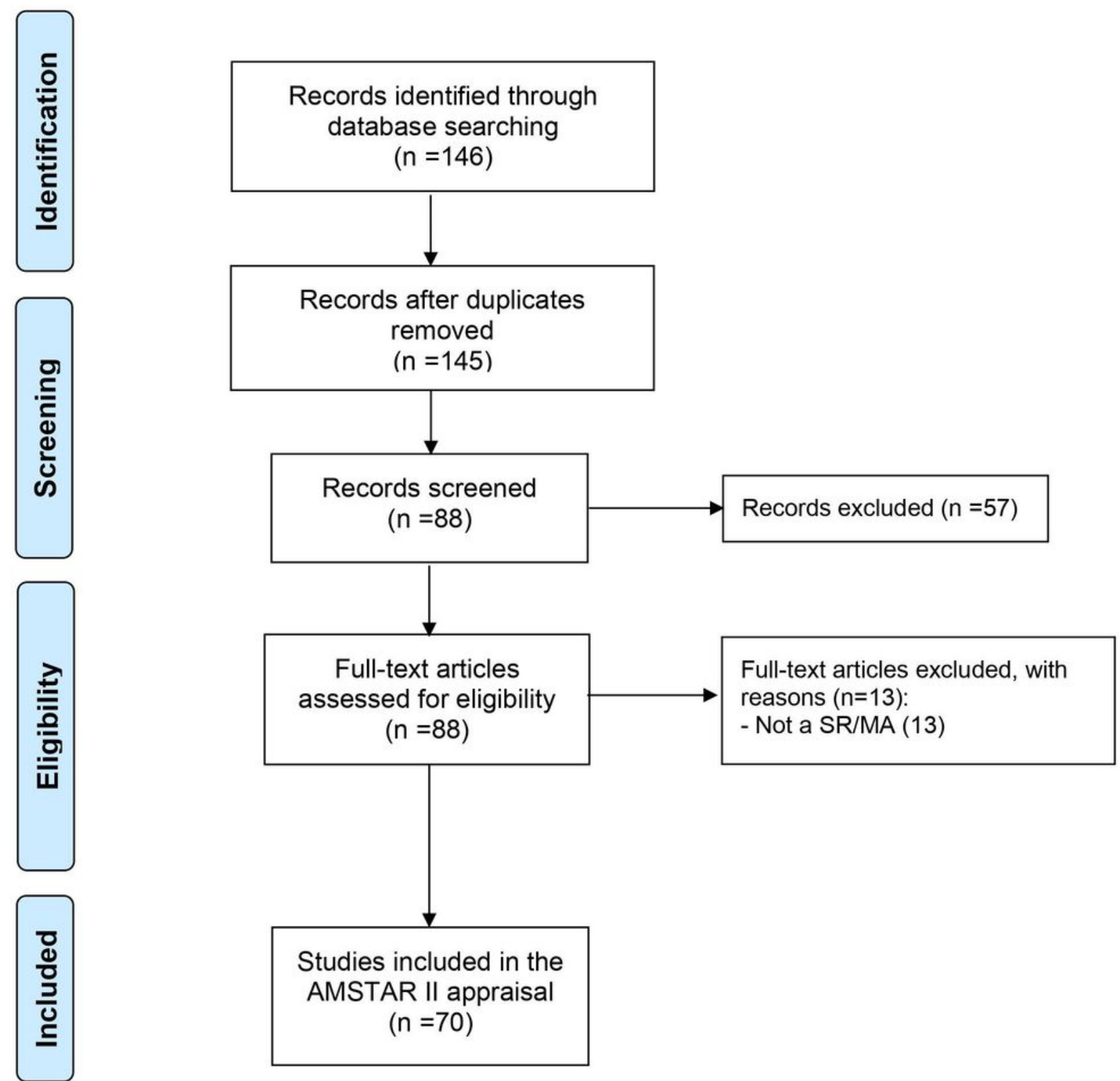

Figure 1. Flowchart showing the search results and reasons for exclusion.

\section{Figure 1}

Flowchart showing the search results and reasons for exclusion 


\section{Total $(n=70)$}

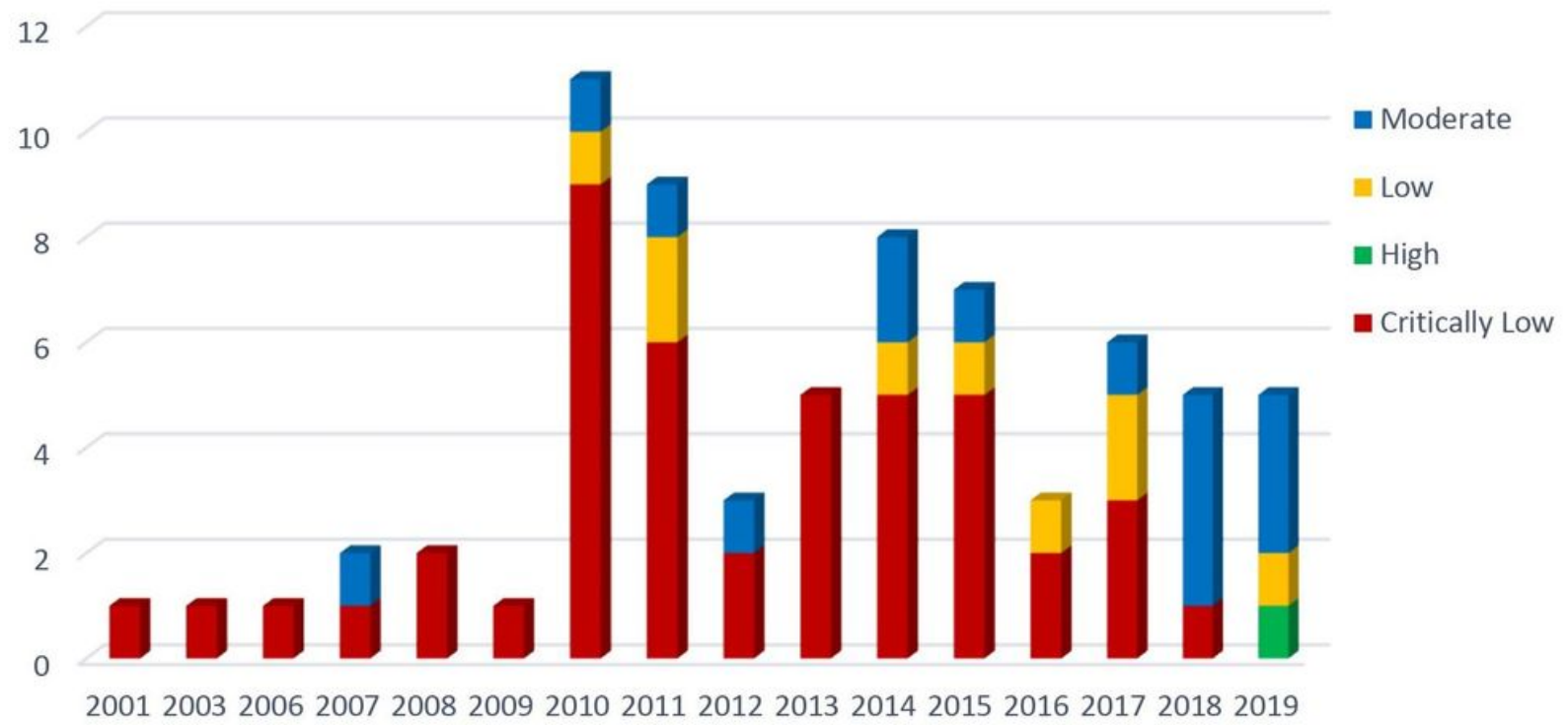

Figure 2. Overall methodological quality score of systematic reviews published between 2001 and 2019.

Figure 2

Overall methodological quality score of systematic reviews published between 2001 and 2019 


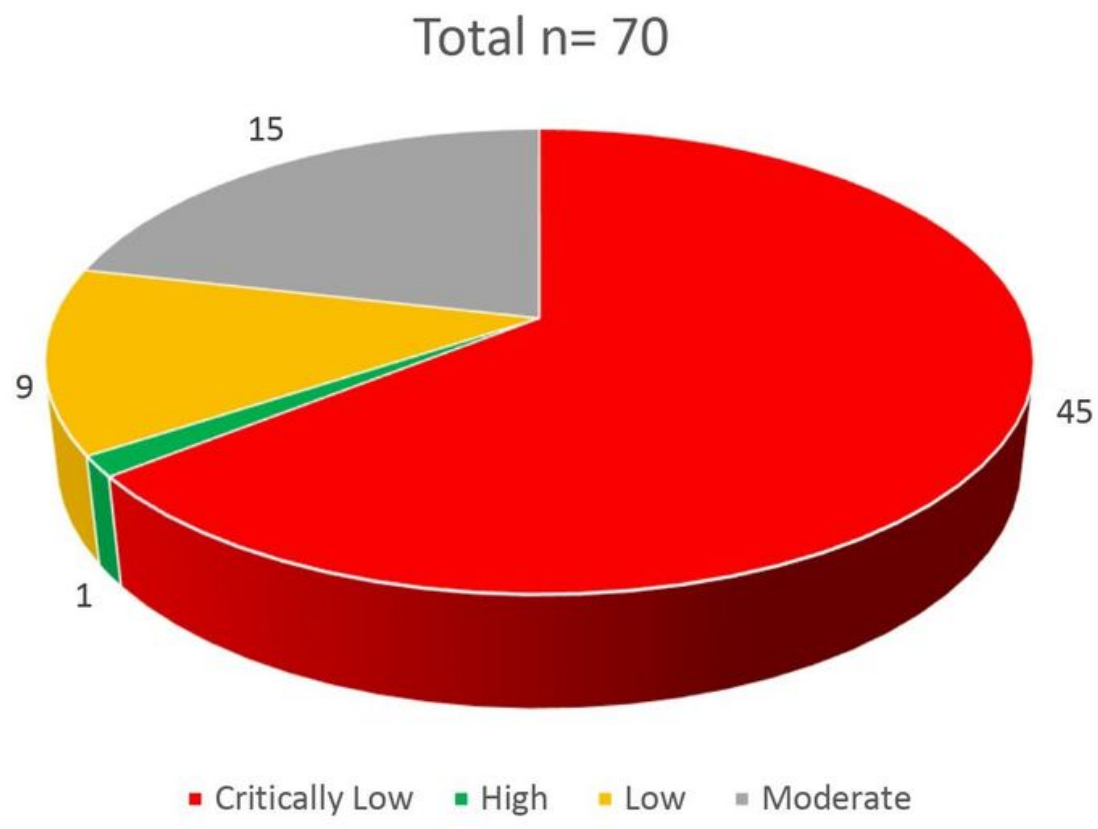

Figure 3. Overall methodological quality score (AMSTAR II) of the included systematic reviews.

\section{Figure 3}

Overall methodological quality score (AMSTAR II) of the included systematic reviews

\section{Supplementary Files}

This is a list of supplementary files associated with this preprint. Click to download.

- Tables.pdf

- Additionalfile1.pdf

- Additionalfile2.pdf 